\title{
Corrigendum: Chemical Stimulants and Stressors Impact the Outcome of Virus Infection and Immune Gene Expression in Honey Bees (Apis mellifera)
}

\author{
Fenali Parekh ${ }^{1,2,3}$, Katie F. Daughenbaugh ${ }^{2,3}$ and Michelle L. Flenniken ${ }^{1,2,3 *}$ \\ ${ }^{1}$ Department of Microbiology and Immunology, Montana State University, Bozeman, MT, United States, \\ 2 Department of Plant Sciences and Plant Pathology, Montana State University, Bozeman, MT, United States, \\ ${ }^{3}$ Pollinator Health Center, Montana State University, Bozeman, MT, United States
}

\section{OPEN ACCESS}

Edited and reviewed by:

Humberto Lanz-Mendoza, National Institute of Public Health, Mexico

*Correspondence: Michelle L. Flenniken michelle.flenniken@montana.edu

Specialty section:

This article was submitted to Comparative Immunology, a section of the journal

Frontiers in Immunology

Received: 28 December 2021 Accepted: 24 January 2022

Published: 02 March 2022

Citation: Parekh F, Daughenbaugh KF and Flenniken ML (2022) Corrigendum: Chemical Stimulants and Stressors

Impact the Outcome of Virus Infection and Immune Gene Expression in Honey Bees (Apis mellifera).

Front. Immunol. 13:844882. doi: 10.3389/fimmu.2022.844882
Keywords: honey bee, Apis mellifera, insect antiviral defense, honey bee viruses, deformed wing virus, thymol, fumagillin, clothianidin

\section{A Corrigendum on:}

Chemical Stimulants and Stressors Impact the Outcome of Virus Infection and Immune Gene Expression in Honey Bees (Apis mellifera)

By Parekh F, Daughenbaugh KF and Flenniken ML (2021). Front. Immunol. 12:747848. doi: 10.3389/fimmu.2021.747848

In the original article, there was a mistake in Figures 1, 3, 4, 5, $\mathbf{6}$ and their respective legends as published. The original figures and figure legends, were mislabeled with " $0.16 \mathrm{ppm}$ thyme oil". They should have been labeled with the correct label " $0.16 \mathrm{ppb}$ thyme oil" in all instances. The correct figures and figure legends appears below.

In the original article, there was a mistake in the Supplementary Material as published. Throughout the Supplementary Material " $0.16 \mathrm{ppm}$ thyme oil" should be corrected to " $0.16 \mathrm{ppb}$ thyme oil" in all instances. The correct files appear below.

In the original article, there was an error. Throughout the text it was mistakenly written that "0.16 ppm thyme oil" was utilized for experiments. It should be written "0.16 ppb thyme oil" for all experiments. The original article has been updated.

In addition, in the original article, there was an error. It was mistakenly written " $0.06 \mathbf{~ p p m}$ thymol" instead of "0.06 ppb thymol": "We estimate that $0.16 \mathrm{ppb}$ thyme oil used in this experiment may contain approximately $0.06 \mathrm{ppb}(60 \mathrm{ppb})$ thymol (37\% thymol in $0.16 \mathrm{ppb}$ thyme oil corresponds to $\sim 60 \mathrm{ppb}) "$.

A correction has been made to 4. Materials and Methods, 4.4. Honey Bee Diet Preparation: "Postinjection, honey bees were housed in modified deli containers for the duration of the study. Bees in the control group were fed 50\% sucrose syrup only, whereas bees in treatment groups were fed sucrose syrup containing one of the following additives: $0.16 \mathrm{ppb}$ thyme oil (Body wonders), Fumagilin- $\mathrm{B}^{\circledR}$, fumagillin dicyclohexyl ammonium (Medivet Pharmaceuticals Ltd.) at manufacturer's recommended dose of 25 ppm or a higher dose of $75 \mathrm{ppm}$, or clothianidin at the field relevant sublethal concentration of $1 \mathrm{ppb}$ or near lethal dose of $10 \mathrm{ppb}(130,195,196)$. Thyme oil contains $10 \%$ - 64\% thymol ( 37\% average) depending on the plant species, geographical sources, and harvest season, which may affect the volatile 
A

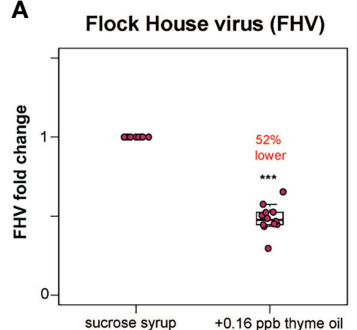

B

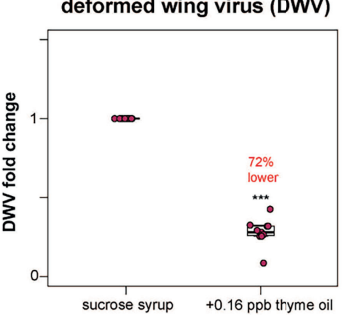

C

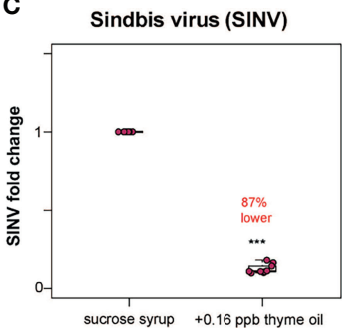

FIGURE 1 | Lower virus abundance in honey bees fed thyme oil augmented sucrose syrup. Virus abundance in individual honey bees that were either fed sucrose syrup only or sucrose syrup augmented with $0.16 \mathrm{ppb}$ thyme oil was assessed at $72 \mathrm{~h}$ post-infection by $\mathrm{gPCR}(\mathrm{n}=9-12$ per treatment group) and the relative virus abundance is presented as ranked fold-changes. Together, these data illustrate that virus-infected bees fed thyme oil augmented sucrose syrup harbored less virus than virus-infected bees fed only sucrose syrup. (A) Flock House virus (FHV)-infected bees fed sucrose syrup augmented with thyme oil (0.16 ppb) had $52 \%$ less FHV (0.48 mean fold change) than bees fed sucrose syrup alone $\left(p=2.1 \times 10^{-5}\right)$. (B) In deformed wing virus (DWV)-infected bees, virus abundance was $72 \%$ less in bees fed sucrose syrup augmented with thyme oil (0.28 mean fold change) compared to bees fed only sucrose syrup ( $p=0.00016)$. (C) Sindbis virus (SINV)-infected bees fed thyme oil augmented sucrose syrup had $87 \%$ less SINV ( 0.13 mean fold change) than bees fed sucrose syrup. Data were analyzed by a pairwise Wilcoxon Rank Sums with a Benjamini-Hochberg correction for multiple comparisons. Asterisks indicate a significant difference in virus abundance; significance levels: ${ }^{\star \star \star} p<$ 0.0005. This figure includes results from one representative biological replicate for each virus (i.e., rep1). The data for all three biological replicates are presented in Supplemental Figure $\mathbf{S 2}$ and raw data are in Supplemental Tables S3, S4.
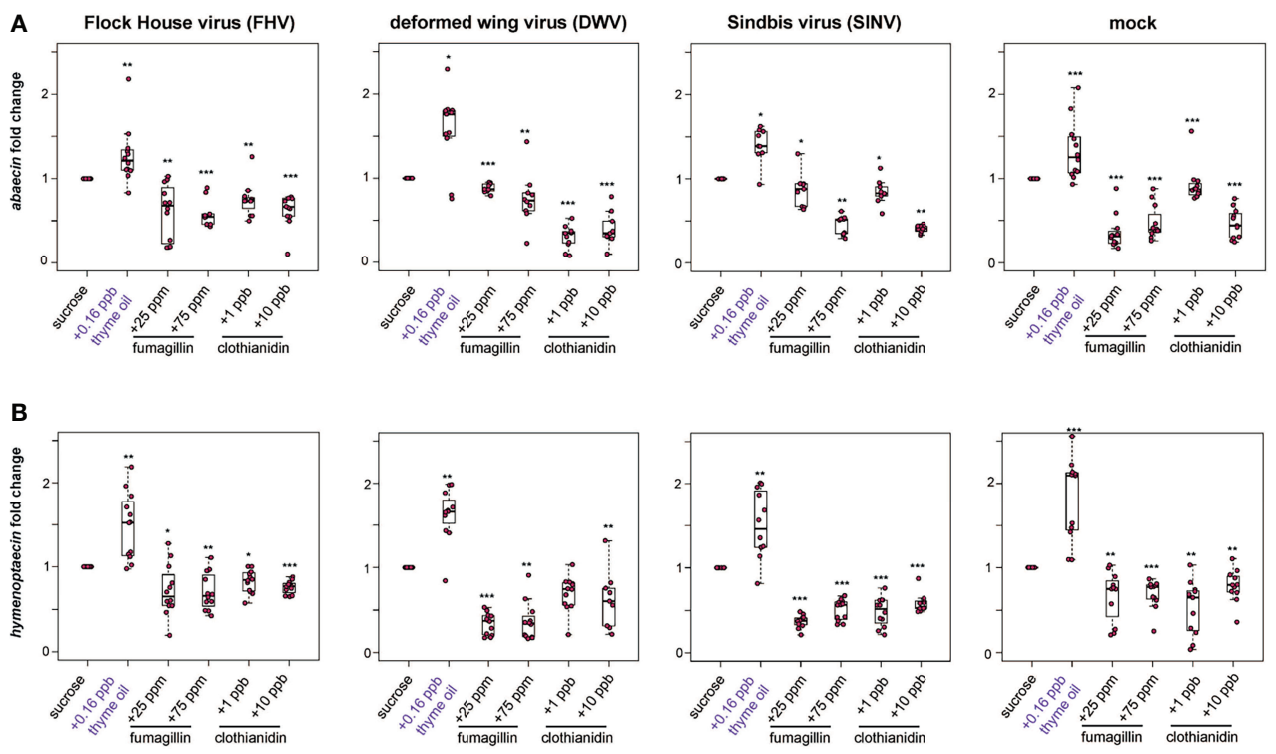

FIGURE 3 | Expression of key RNAi genes was higher in honey bees fed thyme oil augmented sucrose syrup and lower in bees fed sucrose syrup containing fumagillin or clothianidin. Expression of dicer-like and argonaute-2 in honey bees that were either mock- or virus-infected (i.e., FHV, DWV, SINV) and fed sucrose syrup only or syrup containing either thyme oil $(0.16 \mathrm{ppb}$ ), fumagillin $(25 \mathrm{ppm}$ or $75 \mathrm{ppm})$, or clothianidin (1 ppb or $10 \mathrm{ppb})$ was assessed by qPCR. The $\Delta \Delta C t$ method with normalization to rp/8 in mock or virus-infected bees fed sucrose syrup diet was utilized to determine the relative gene expression. (A) Dicer-like (dcr-like) expression was higher in virus-infected (i.e., FHV, DWV, SINV) and mock-infected bees fed thyme oil containing sucrose syrup, and lower in bees fed sucrose syrup containing fumagillin (25 ppm or $75 \mathrm{ppm}$ ) or clothianidin (1 ppb or $10 \mathrm{ppb}$ ) relative to bees fed sucrose syrup. (B) Argonaute-2 (ago2) expression was higher in virus infected (i.e., FHV, DWV, SINV) or mock-infected bees fed thyme oil augmented sucrose syrup and reduced in bees fed diets containing fumagillin (25 ppm or 75 ppm) or clothianidin (1 ppb or 10 ppb), except in mock-infected bees fed 75 ppm fumagillin, ago2 expression was similar to expression levels in bees fed sucrose only. Data were analyzed by a pairwise Wilcoxon Rank Sums with a Benjamini-Hochberg correction for multiple comparisons. Asterisks indicate a significant change in gene expression compared to sucrose only control; significance levels: ${ }^{\star} p<0.05 ;{ }^{\star *} p<0.005 ;{ }^{\star \star *} p<0.0005$. This figure shows representative biological replicate for each gene (i.e., rep2 for dcr-like and rep3 for ago2). The data for all three biological replicates are presented in Supplemental Figures S4, S5. Raw data are included in Supplemental Tables S7-S10. 

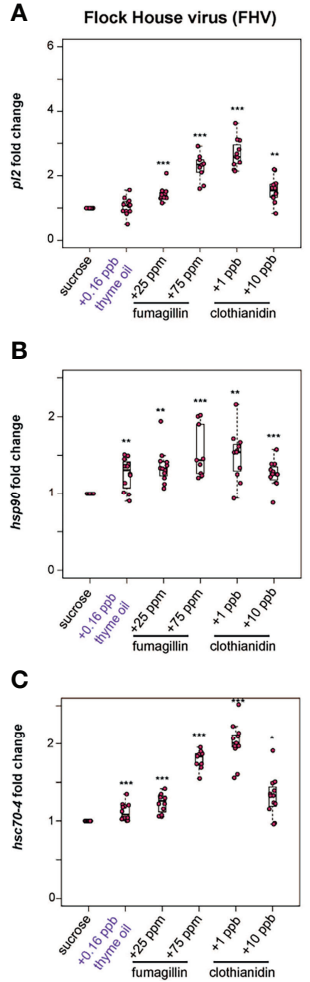

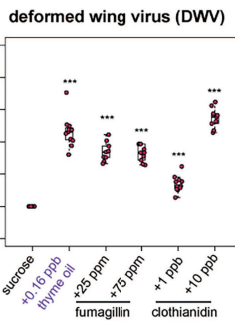

Sindbis virus (SINV)
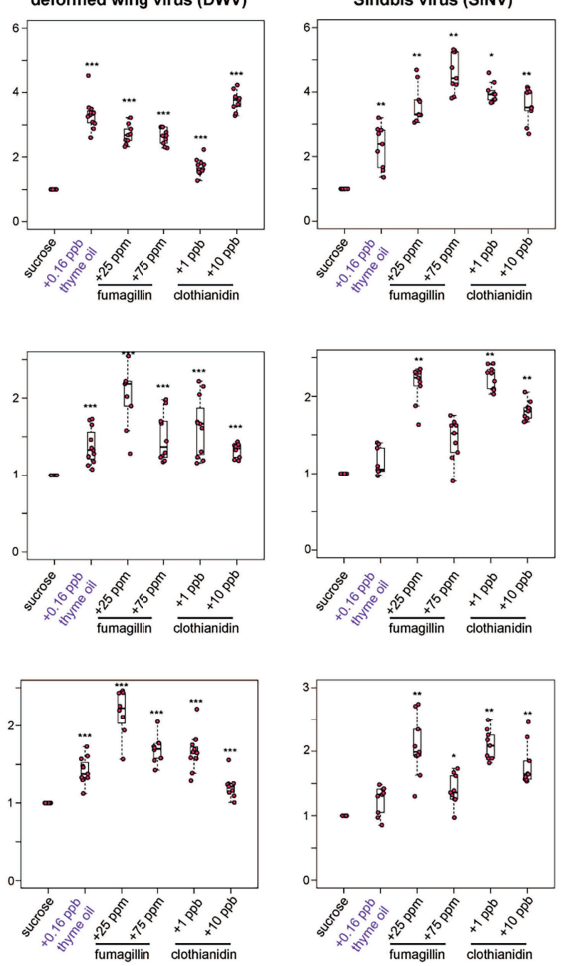
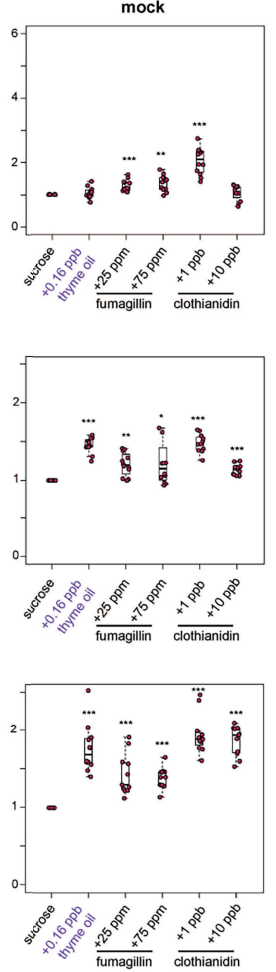

FIGURE 4 | Expression of heat shock protein encoding genes was higher in honey bees fed sucrose syrup containing thyme oil, fumagillin, or clothianidin. The relative expression of three genes encoding heat shock proteins (p/2, hsp90, hsc70-4) was assessed using qPCR in mock or virus-infected bees fed sucrose syrup only or sucrose syrup containing additivities (i.e., thyme oil, fumagillin, or clothianidin). The $\Delta \Delta \mathrm{Ct}$ method with normalization to rp/8 in mock or virus-infected bees fed sucrose syrup diet was utilized to determine the relative gene expression. (A) Protein lethal(2)essential for life-like (p/2) expression in virus-infected bees (i.e., FHV, DWV, SINV) fed sucrose syrup containing thyme oil (0.16 ppb), fumagillin (25 ppm or $75 \mathrm{ppm}$ ) or clothianidin (1 ppb or $10 \mathrm{ppb})$ was higher compared to expression in bees fed sucrose only. In mock-infected bees, pl2 expression in bees fed sucrose syrup containing stimulant (0.16 ppb thyme oil) or 10 ppb clothianidin was similar to expression levels in bees fed sucrose only, whereas pl2 expression was higher in bees fed sucrose syrup containing fumagillin $(25 \mathrm{ppm}$ or $75 \mathrm{ppm}$ ) or 1 ppb clothianidin. (B) Heat shock protein 90 (hsp90) expression was higher in majority of the treatment groups, including virus- or mock-infected infected bees fed augmented sucrose syrups compared to bees fed non-augmented sucrose syrup except in SINV-infected bees fed thyme oil or 75 ppm fumagillin, which had similar $h s p 90$ expression levels to bees fed sucrose only. (C) Heat shock 70-kDa protein cognate 4 (hsc70-4) expression was higher in the majority of treatment groups including mock and virus-infected bees fed sucrose syrup containing either thyme oil $(0.16 \mathrm{ppb})$, fumagillin $(25 \mathrm{ppm}$ or $75 \mathrm{ppm}$ ) or clothianidin (1 ppb or $10 \mathrm{ppb}$ ), except in SINV-infected bees fed sucrose syrup containing thyme oil, which had hsc70-4 expression levels

similar to the controls. Data were analyzed by a pairwise Wilcoxon Rank Sums with a Benjamini-Hochberg correction for multiple comparisons. Asterisks indicate a significant change in gene expression compared to sucrose only control; significance levels: ${ }^{*} p<0.05 ;{ }^{* *} p<0.005 ;{ }^{* \star *} p<0.0005$. This figure shows representative biological replicate for p/2 expression (i.e., rep1). The data for all three biological replicates for $p / 2$ are presented in Supplemental Figure $\mathbf{S 6}$ and raw data are included in Supplemental Tables S11-S16. 


\section{A Flock House virus (FHV)}

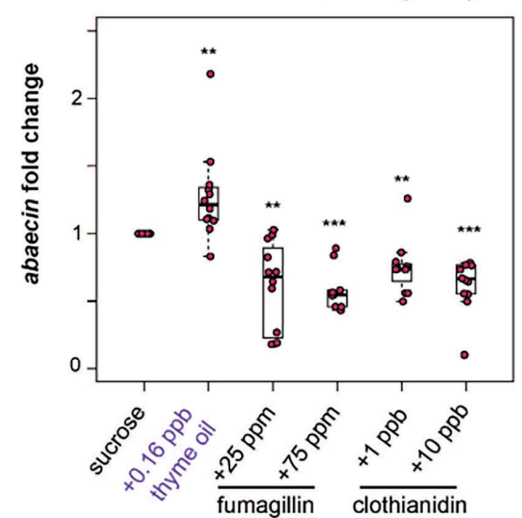

B

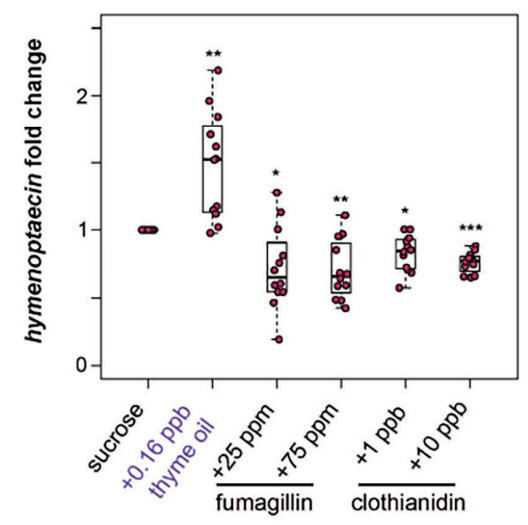

deformed wing virus (DWV)
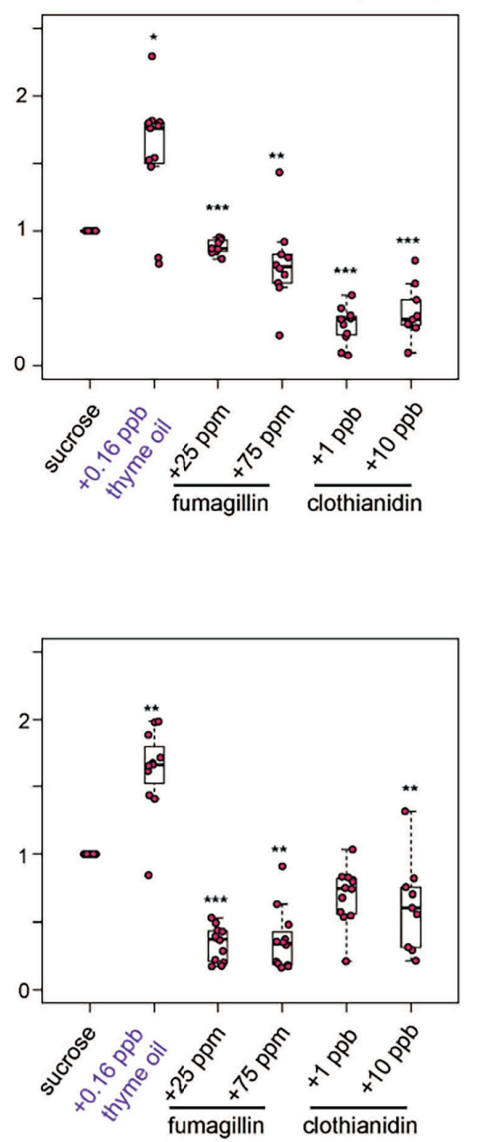

Sindbis virus (SINV)
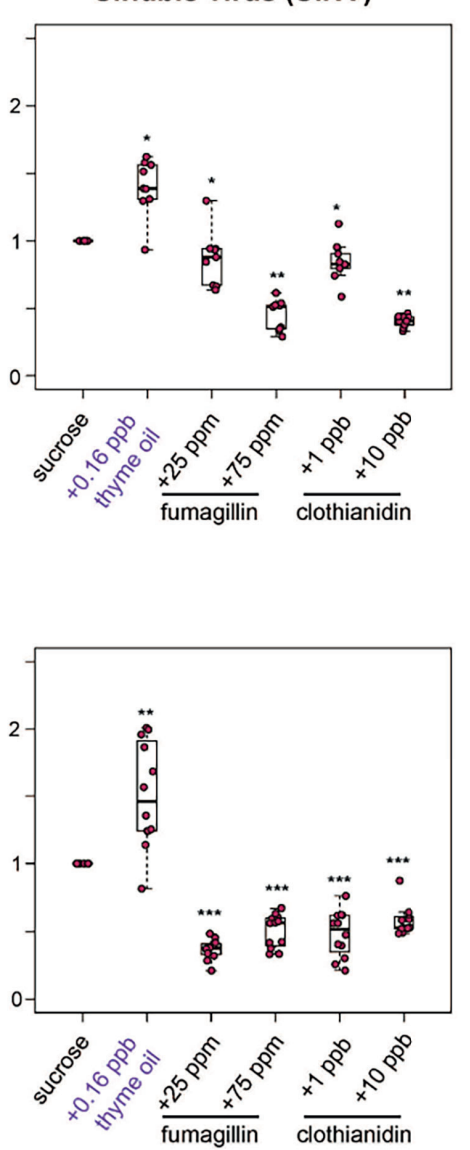

mock
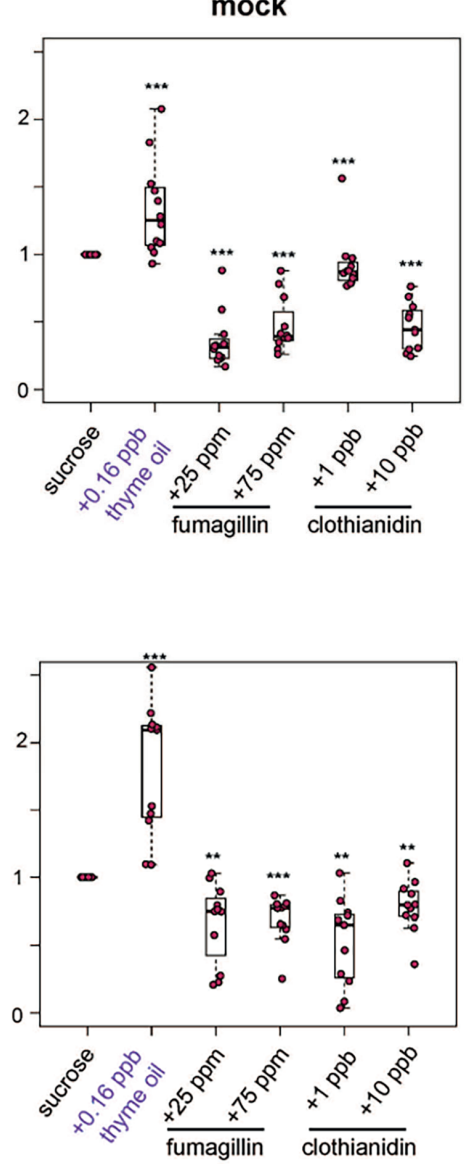

FIGURE 5 | Expression of antimicrobial peptides encoding genes was greater in bees fed thyme oil augmented sucrose syrup and lower in bees fed fumagillin or clothianidin containing sucrose syrup. The expression of two antimicrobial peptides (abaecin and hymenoptaecin) was assessed in mock and virus-infected bees fed sucrose syrup containing additives. (A) In virus- and mock-infected bees (i.e., FHV, DWV, SINV), abaecin expression was higher in bees fed sucrose syrup containing thyme oil and lower in bees fed sucrose syrup containing fumagillin (25 ppm or $75 \mathrm{ppm}$ ) or clothianidin (1 ppb or $10 \mathrm{ppb}$ ) compared to bees fed sucrose only. (B) In virus-infected bees (i.e., FHV, DWV, SINV), hymenoptaecin expression was higher in bees fed sucrose syrup containing thyme oil and lower in bees fed sucrose syrup containing fumagillin (25 ppm or 75 ppm) or clothianidin (1 ppb or $10 \mathrm{ppb).} \mathrm{Hymenoptaecin} \mathrm{expression} \mathrm{was} \mathrm{higher} \mathrm{in} \mathrm{mock-infected} \mathrm{bees} \mathrm{fed} \mathrm{sucrose} \mathrm{syrup} \mathrm{augmented} \mathrm{with} 0.16$ ppb thyme oil, but lower in mock-infected bees fed either fumagillin (i.e., 25 ppm or 75 ppm) or clothianidin (1 ppb or $10 \mathrm{ppb}$ ) containing sucrose syrup. Data were analyzed by a pairwise Wilcoxon Rank Sums with a Benjamini-Hochberg correction for multiple comparisons. Asterisks indicate a significant change in gene expression compared to sucrose only control; significance levels: ${ }^{*} p<0.05$; ${ }^{* *} p<0.005 ;{ }^{* \star *} p<0.0005$. This figure shows representative biological replicate for the expression of each gene (i.e., rep1 for abaecin and rep3 for hymenoptaecin). The data for one additional biological replicate is presented in Supplemental Figures S7, S8. Raw data are included in Supplemental Tables S17-S20. 

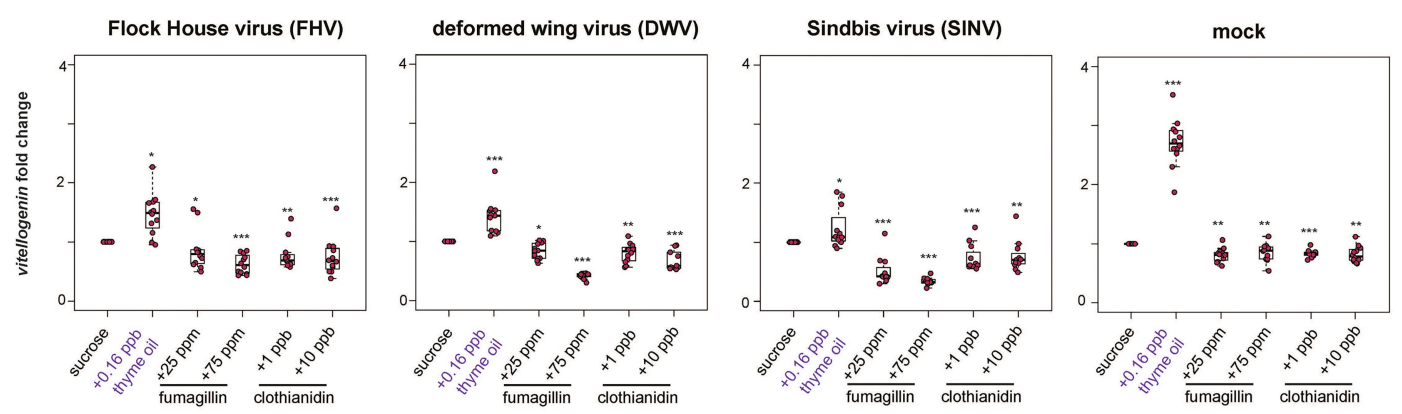

FIGURE 6 | Vitellogenin expression was higher in bees fed sucrose syrup containing thyme oil and lower in bees fed sucrose syrup containing fumagillin or clothianidin. Vitellogenin expression was assessed using $\mathrm{qPCR}$ and the relative gene expression was analyzed using the $\Delta \Delta \mathrm{Ct}$ method with normalization to rp/8 and relative to expression in mock or virus-infected bees fed sucrose syrup only. In virus-infected (i.e., FHV, DWV, SINV) and mock-infected honey bees vitellogenin expression was greater in bees fed sucrose syrup containing thyme oil 0.16 ppb and reduced in bees fed sucrose syrup containing fumagillin ( 25 ppm or 75 ppm) or clothianidin (1 ppb or $10 \mathrm{ppb}$ ) relative to sucrose only fed bees. Asterisks indicate a significant change in gene expression compared to sucrose only control; significance levels: ${ }^{\star} p<0.05 ;{ }^{\star \star} p<0.005 ;{ }^{* \star} p<0.0005$. This figure shows representative biological replicate for vitellogenin expression. The data for one additional biological replicate is presented in Supplemental Figure S9. Raw data are included in Supplemental Tables S21, S22.

composition of the plant $(113,116-118)$. We estimate that $0.16 \mathrm{ppb}$ thyme oil used in this experiment may contain approximately 0.06 $\mathrm{ppb}$ thymol (37\% thymol in $0.16 \mathrm{ppb}$ thyme oil corresponds to $0.06 \mathrm{ppb})$. For clothianidin treatments we utilized the commercially available Poncho ${ }^{\circledR} 600$, which contains $48 \%$ of the active ingredient clothianidin. A working stock of 1000 ppb clothianidin was prepared by $1: 10$ serial dilutions in $50 \%$ sucrose solution, which was further diluted in $50 \%$ sucrose to prepare $1 \mathrm{ppb}$ (i.e., $10 \mathrm{ul}$ of $1000 \mathrm{ppb}$ Poncho ${ }^{\circledR}$ 600) and $10 \mathrm{ppb}$ (i.e., 100 ul of $1000 \mathrm{ppb}$ Poncho ${ }^{\circledR}$ 600) clothianidin solutions. Honey bees were fed sucrose solution either alone or with additives using cage feeders that were made by putting two holes on each side of a $1.5 \mathrm{~mL}$ centrifuge tube; sucrose solution was checked daily and refilled as needed throughout the study."

The authors apologize for these errors and state they do not change the scientific conclusions of the article in any way.

\section{SUPPLEMENTARY MATERIAL}

The Supplementary Material for this article can be found online at: https://www.frontiersin.org/articles/10.3389/fimmu.2022.844882/ full\#supplementary-material

Publisher's Note: All claims expressed in this article are solely those of the authors and do not necessarily represent those of their affiliated organizations, or those of the publisher, the editors and the reviewers. Any product that may be evaluated in this article, or claim that may be made by its manufacturer, is not guaranteed or endorsed by the publisher.

Copyright (c) 2022 Parekh, Daughenbaugh and Flenniken. This is an open-access article distributed under the terms of the Creative Commons Attribution License (CC BY). The use, distribution or reproduction in other forums is permitted, provided the original author(s) and the copyright owner(s) are credited and that the original publication in this journal is cited, in accordance with accepted academic practice. No use, distribution or reproduction is permitted which does not comply with these terms. 